\title{
A Fusion Algorithm for Visible and Infrared Images Based on Region Growing Method
}

\author{
Xinming Zhou \\ The Chinese People’s Liberation Army Unit 91550, Dalian, 116023, China \\ Email: zhouXM_DL@163.com
}

Keywords: Target tracking; object segmentation; image registration; region growing

\begin{abstract}
Aiming at the real-time problem of video sequence fusion in infrared and visible target tracking, the target region is extracted from the infrared image by region growing method based on the gray-level difference between the target and the background in infrared image and the region growing method commonly used in target segmentation in target tracking. The outer target region is fused with the background region of the visible image which has been registered by the image, and the fused image with the good target indication characteristic of the infrared image and the clear scene information of the visible image is finally obtained.
\end{abstract}

\section{Introduction}

Infrared imaging sensor and visible light imaging sensor are two commonly used sensors, which are widely used in aviation, navigation and other military fields of detection, monitoring and tracking [1]. Because of their different imaging principles, their performance varies. Infrared imaging sensors recognize targets by detecting the difference of thermal radiation between the target and the background, so they have a special ability of recognition and camouflage. However, they are insensitive to the brightness changes of the scene and have low imaging clarity, which is not conducive to eye interpretation [2]. The visible light imaging sensor is sensitive to the reflection of the target scene, and the acquired image has high definition, which can provide the detailed information of the scene where the target is located. Therefore, the fusion of infrared and visible images will be beneficial to synthesize better target indication characteristics of infrared images and clear scene information of visible images.

The fusion of still images has been extensively studied, but the fusion algorithms used in static image fusion algorithms are more complex and require a lot of computing time, which can not meet the real-time requirements of video sequence processing [3]. Zhao Peng et al. proposed a feature level video sequence fusion method based on dynamic contour feature search in document [4]. However, this method needs newmark iteration, and the calculation of the algorithm is more complex. John J. Lewis et al. compared pixel-based and region-based image fusion in literature [5], and showed that region-based fusion is superior to pixel-based fusion. In this paper, the target region of infrared image is fused with the clear background region of visible light image by using the region growing segmentation method which is commonly used in target detection. Experiments show that the proposed algorithm can not only obtain a more clear and informative fusion image than other commonly used fusion algorithms, but also combine the fusion algorithm with the image tracking algorithm, which saves a lot of time for target tracking of fused video sequence images, thus guaranteeing the real-time performance of the whole tracking algorithm.

\section{Region growing method}

Region growing is a serial segmentation technique based on region [6]. Its basic idea is to assemble pixels with similar properties to form a region. First, a seed pixel is found as the starting point of growth in each region to be segmented, and then the neighborhood pixels with the same or similar properties as the seed pixels (according to some pre-determined growth or similarity criteria) 
are merged into the region where the seed pixels are located [7]. These new pixels are treated as new seed pixels to continue the above process until no more pixels satisfying the conditions can be included, so that a region is grown.

The selection of seed pixels often depends on the characteristics of specific problems. When detecting targets in military infrared images [8], the brightest pixels in the image can be selected as seed pixels because the target radiation is large. If there is no prior knowledge about the specific problem, each pixel can be calculated by the growth criteria. If the result is clustering, the pixels close to the cluster center can be regarded as seed pixels. The selection of growth criterion does not depend on the specific problem itself, but on the data of the image used.

\section{Fusion algorithm based on region growing}

If the absolute value of the difference subtracted from 1.0 by the ratio of gray values between adjacent pixels is less than the threshold value, the growth criterion can be considered to be satisfied. Knowing that the region boundary is usually formed by pixels with abrupt change in pixel gray level, the effective gradient value of image pixel refers to that a pixel in the horizontal or vertical direction of the image changes more than a certain effective value when the pixel there is a sudden change, not belonging to the category of pixels around it. In order to reduce the judgment error, this paper uses the absolute value of the ratio of the effective gradient value of the pixel to the gray value of the pixel $G_{f}(x, y)$ to determine whether the pixel is a gray mutation point.

$$
G_{f}(x, y)=\left\{\begin{array}{l}
\text { IWidth }-1 \\
\sum_{m=0} \sum_{n=0}^{\text {lHeight }-1}\left|1.0-\frac{f(m, n-1)+f(m-1, n)+f(m+1, n)+f(m, n+1)}{4 f(m, n)}\right|
\end{array}\right.
$$

Note that $\mathrm{M}$ is initialized to zero for the number of effective gray mutation points. Traversing the whole infrared image, when the pixel and its 4 neighbors $G_{f}(x, y)$ are greater than 0.1 , the pixel is considered as a gray level mutation point, M plus 1.

The average value $G_{f}(x, y)$ of all effective mutations in infrared images is:

$$
\text { Threhold }=\frac{G_{f}(x, y)}{M}
$$

Because some regions in the region extracted by region growth may be between the maximum target gray area and the minimum background gray area, and these regions may be other targets that we need to deal with later, or may be the image of the surrounding background objects, it is necessary to discriminate and classify these regions. Firstly, the region pixel mean value is calculated for each region extracted by Region growing, and the number of the region pixel mean value from small to large is listed as Emin, Emin $+1, \ldots$ Emax, then use membership function:

$$
\mu_{i}=\frac{E_{i}-E_{\min }}{E_{\max }-E_{\min }}, E_{i} \in\left[E_{\min }, E_{\max }\right]
$$

The fusion image is obtained by replacing the pixel value of the corresponding position in the matched visible light image with the pixel value of the target region of the infrared image after classification and discrimination. This method can also be applied to image fusion of low frequency layers in Pyramid decomposition.

\section{Experimental results and evaluation}

The processing procedure and feasibility of the algorithm are illustrated by two sets of experimental images. The algorithm is compared with Laplacian pyramid fusion, contrast pyramid fusion, wavelet decomposition fusion and principal component analysis weighting coefficient fusion, which are commonly used in image fusion. The subjective evaluation and objective 
evaluation of the fusion are given. The advantage of the algorithm is obtained. The evaluation parameters of the objective evaluation criteria are cross entropy, standard deviation and grey mean value. The cross entropy can measure the information difference between two images. The smaller the cross entropy is, the smaller the difference between images is, that is, the better the fusion effect is. The standard deviation reflects the discrete degree of the image gray distribution, and the high contrast image corresponds to the large standard deviation, and vice versa. The average gray value reflects the average brightness that the human eye feels. If the average gray value of the image is moderate, the subjective effect of the image will be better.

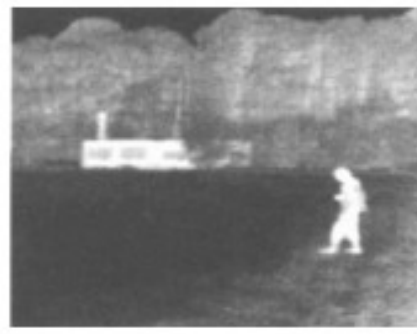

(a)

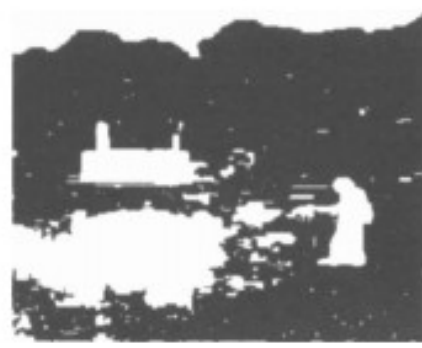

(d)

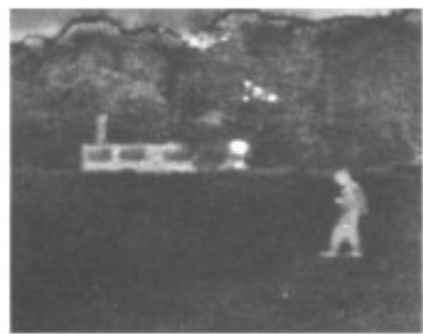

(g)

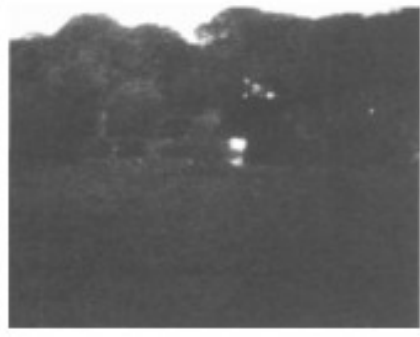

(b)

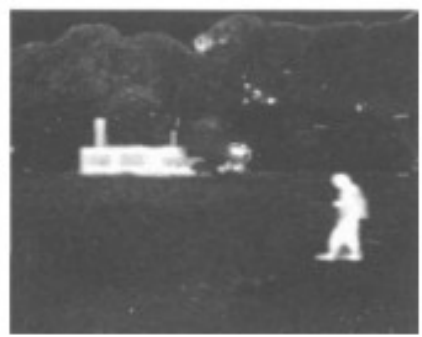

(e)

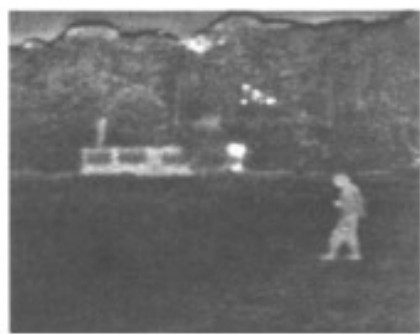

(h)

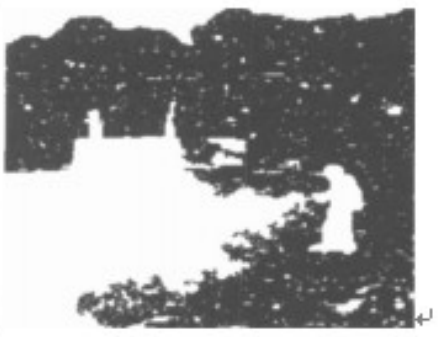

(c)

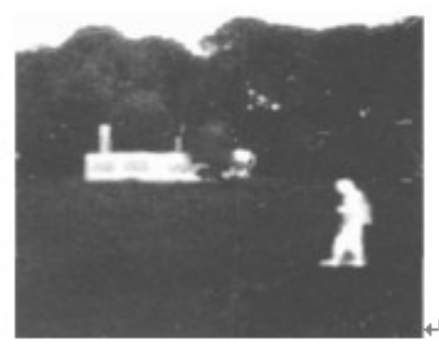

(f)

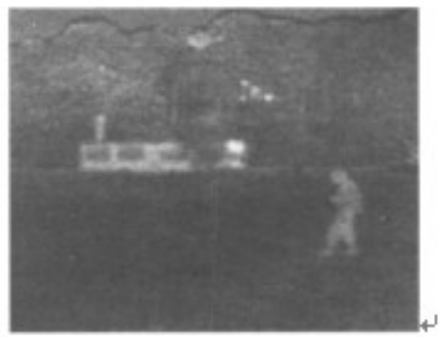

(i)

Fig.1. The results were processed by region growing, classification and fusion

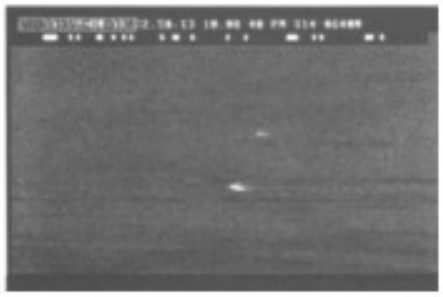

(a)

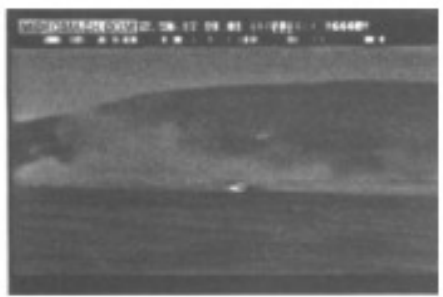

(d)

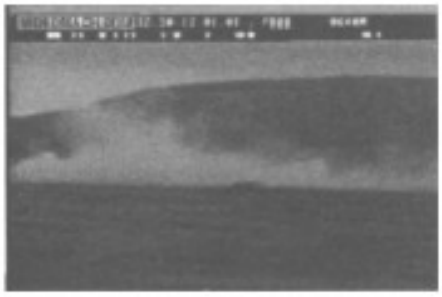

(b)

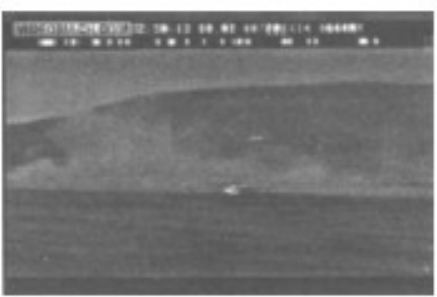

(e)

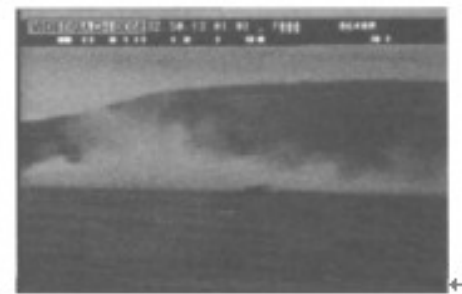

(c)

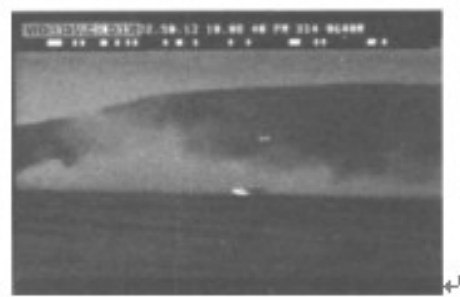

(f)

Fig.2. Image and processing result to be fused 
In Figure 1, (a) and (b) are the original infrared and visible images to be fused; (c) are the results of region growing and segmentation of the infrared image without filtering; (d) are the results of region growing and segmentation of the infrared image after filtering and denoising; (e) are the fused images obtained by classifying and distinguishing the undivided regions; (f) are the results of region growing and segmentation of the infrared image after filtering and denoising; (f) are the fused images obtained by classifying and distinguishing the undivid The fused images are obtained by classifying and discriminating the segmented regions. (g), (h), (i) The fused images are obtained by using Laplace pyramid fusion, wavelet decomposition fusion and principal component analysis weighted coefficient fusion respectively.

In Figure 2, (a) and (b) are infrared and visible images to be fused; (c), (d), (e) are fused images obtained by Laplacian pyramid fusion, contrast pyramid fusion and wavelet decomposition fusion, respectively; (f) are fused images obtained by our algorithm. Comparing the effect of image (f) from subjective vision, it is found that the fusion image obtained by the other three algorithms is superior to the fusion image.

\section{Conclusion}

By combining the region growing commonly used in target segmentation with region-based image fusion, a video fusion method for multi-sensor target tracking is proposed. The algorithm is improved on the premise of the traditional region growing method, which enhances the ability of object extraction and classification. Experiments show that the algorithm not only has less computation time, but also gets better fusion results than the common pixel-level fusion algorithm.

\section{References}

[1] JOHN J L, ROBERT J Ocallaghan, STAVRIGN,etal. Pixel and region-base image fusion with complex wavelets [J].Elsevier, 2017(8):119-130.

[2] Mahesh B. Chappalli, N. K. Bose. Enhanced Biggs-Andrews asymmetric iterative blind deconvolution [J]. Multidim Syst Sign Process, 2016, 17:151-175.

[3] Jiang Ming, Wang Ge. Development of blind image deconvolution and its applications [J]. Journal of X-Ray Science and Technology, 2013, 11:13-19.

[4] Chen S.D, Ramli A.R. Minimum mean brightness error histogram equalization in contrast enhancement [J]. IEEE Transactions on Consumer Electronics, 2013, 49(4): 1310-1319.

[5] Chen S.D, Ramli A.R. Contrast enhancement using recursive mean-separate histogram equalization for scalable brightness preservation [J]. IEEE Transactions on Consumer Electronics, 2013, 49(4): 1301-1309.

[6] Kim J.Y, Kim L.S. An advanced contrast enhancement using partially overlapped sub-block histogram equalization [J]. IEEE Transactions on Circuits and Systems, 2016, 11(4): 475-484.

[7] Wong A, Clausid A, Fieguth P. Adaptive Monte Carlo Retinex method for illumination and reflectance separation and color image enhancement[C]. Proc of Canadian Conference on Computer and Robot Vision. Washington DC: IEEE Computer Society, 2016: 108-115.

[8] Caselles V, Lisani J. L. Shape preserving local histogram modification [J]. IEEE Trans Image Process, 2010, 8(2):220-230. 